University of Nebraska - Lincoln

DigitalCommons@University of Nebraska - Lincoln

Timothy J. Gay Publications

Research Papers in Physics and Astronomy

November 1977

\title{
Alignment of helium excited by thin carbon foils
}

\author{
R. D. Hight \\ University of Toledo, Toledo, Ohio \\ R. M. Schectman \\ University of Toledo, Toledo, Ohio \\ H. G. Berry \\ University of Chicago, Chicago, Illinois \\ G. Gabrielse \\ University of Chicago, Chicago, Illinois \\ Timothy J. Gay \\ University of Nebraska - Lincoln, tgay1@unl.edu
}

Follow this and additional works at: https://digitalcommons.unl.edu/physicsgay

Part of the Physics Commons

Hight, R. D.; Schectman, R. M.; Berry, H. G.; Gabrielse, G.; and Gay, Timothy J. , "Alignment of helium excited by thin carbon foils" (1977). Timothy J. Gay Publications. 4.

https://digitalcommons.unl.edu/physicsgay/4

This Article is brought to you for free and open access by the Research Papers in Physics and Astronomy at DigitalCommons@University of Nebraska - Lincoln. It has been accepted for inclusion in Timothy J. Gay Publications by an authorized administrator of DigitalCommons@University of Nebraska - Lincoln. 
Phys. Rev. A 16, 1805 - 1810 (1977) [Issue 5 - November 1977]

\section{Alignment of helium excited by thin carbon foils}

R. D. Hight and R. M. Schectman

Department of Physics and Astronomy, University of Toledo, Toledo, Ohio 43606

H. G. Berry

Department of Physics, University of Chicago, Chicago, Illinois 60637 and Argonne National Laboratory, Argonne, Illinois 60439

G. Gabrielse and T. Gay

Department of Physics, University of Chicago, Chicago, Illinois 60637

Received 7 July 1977

We have measured the linear polarization of the 5016- $\AA, 2 s{ }^{1} S-3 p{ }^{1} P$ transition of Hei excited by a thin carbon foil perpendicular to the incident $\mathrm{He}^{+}$beam at ion energies $50-500 \mathrm{keV}$. We find that the alignment of the $3 p{ }^{1} P$ term depends upon the beam current density within the range (2$150 \mu \mathrm{A} \mathrm{cm}^{-2}$ ) measured. Oscillations in the dependence of both $M / I$ (the linear polarization fraction) upon energy and the current density variation of $M / I$ with energy are observed. We discuss possible origins of these variations.

(C)1977 The American Physical Society

URL: http://link.aps.org/abstract/PRA/v16/p1805

DOI: 10.1103/PhysRevA.16.1805 


\title{
Alignment of helium excited by thin carbon foils*
}

\author{
R. D. Hight and R. M. Schectman \\ Department of Physics and Astronomy, University of Toledo, Toledo, Ohio 43606 \\ H. G. Berry \\ Department of Physics, University of Chicago, Chicago, Illinois 60637 \\ and Argonne National Laboratory, Argonne, Illinois 60439 \\ G. Gabrielse and T. Gay \\ Department of Physics, University of Chicago, Chicago, Illinois 60637 \\ (Received 7 July 1977)
}

\begin{abstract}
We have measured the linear polarization of the 5016- $\AA, 2 s{ }^{1} S-3 p{ }^{1} P$ transition of $\mathrm{He}_{1}$ excited by a thin carbon foil perpendicular to the incident $\mathrm{He}^{+}$beam at ion energies $50-500 \mathrm{keV}$. We find that the alignment of the $3 p{ }^{1} P$ term depends upon the beam current density within the range $\left(2-150 \mu \mathrm{Acm}^{-2}\right)$ measured. Oscillations in the dependence of both $M / I$ (the linear polarization fraction) upon energy and the current density variation of $M / I$ with energy are observed. We discuss possible origins of these variations.
\end{abstract}

\section{INTRODUCTION}

The excitation of atoms traversing thin foils has for some time proved to be a most useful tool in the study of atomic structure. ${ }^{1}$ Nonetheless, very little progress has been made toward understanding the details of the excitation process itself. Thus, despite the fact that alignment of atoms traversing the foil has been utilized in fine and hyperfine structure studies for a number of years, ${ }^{1,2}$ even the origin of this alignment has not yet been clearly es tablished. $\backslash$ The relative roles of bulk excitation and surface excitation ${ }^{3}$ have not clearly been delineated; neither has the energy dependence of the alignment produced been mapped in detail as a function of incident beam energy except in one or two isolated examples. ${ }^{4}$ The importance of surface interactions was emphasized by a recent series of experiments with tilted foils. ${ }^{5}$ Attempts to understand these results theoretically in terms of a bulk effect modified by a surface electric field, ${ }^{6}$ interactions at the surface, ${ }^{7}$ geometrical effects, ${ }^{8}$ and electron pickup ${ }^{9}$ have all failed to agree with observation.

Ions excited by passage through perpendicular foils possess cylindrical symmetry of excitation and the dipole radiation emitted can then be characterized by just two parameters for each excited state: the total light yield and a single alignment parameter. In an effort to clarify the foil excitation mechanism, we have made measurements in this simpler geometry. Variations of the light yield and alignment with foils of different materials have been observed and are described in a separate paper. ${ }^{10}$ We show here that the alignment produced depends upon the beam current density in a relatively complicated energy-dependent fashion. Thus any theoretical model to explain the origin of the alignment produced in the ion-foil interaction must also explain this surprising feature.

\section{EXPERIMENT}

Determination of the alignment produced by the ion-foil interaction was carried out by measuring the linear polarization fraction (relative Stokes parameter ${ }^{2}$ ) $M / I$ of the light emitted perpendicular to the beam direction, where

$$
M / I=\left(I_{\|}-I_{\perp}\right) /\left(I_{\|}+I_{\perp}\right) .
$$

$I_{\|}$and $I_{\perp}$ are the light intensities with polarization vector parallel and perpendicular to the beam axis, respectively. For a perpendicular foil and viewing perpendicular to the beam axis, $M / I$ is specified ${ }^{11}$ in terms of the alignment parameter $A_{0}^{\text {col }}$, and for the $2 s{ }^{1} S-3 p{ }^{1} P$ transition is

$$
M / I=-3 A_{0}^{\mathrm{col}} /\left(2-A_{0}^{\mathrm{col}}\right) \text {, }
$$

where

$$
A_{0}^{\text {col }}=\left\langle 3 L_{\|}^{2}-L^{2}\right\rangle / L(L+1) .
$$

Polarization measurements were carried out primarily for the $2 s{ }^{1} S-3 p{ }^{1} P$ transition in He I for incident $\mathrm{He}^{+}$energies ranging from 50 to $500 \mathrm{keV}$; the energy range $60-180 \mathrm{keV}$ was investigated using the University of Chicago accelerator, the range $100-425 \mathrm{keV}$ using the University of Toledo Van de Graaff accelerator, and higher energies with the Argonne National Laboratory dynamitron. Polarization measurements were carried out in Chicago and Argonne using a retardation plate rotated by a stepping motor followed by a fixed linear polarizer, ${ }^{12}$ and in Toledo using a similar system employing a rotating polaroid. ${ }^{5}$ Foil holders of markedly different mechanical, electrical, and 


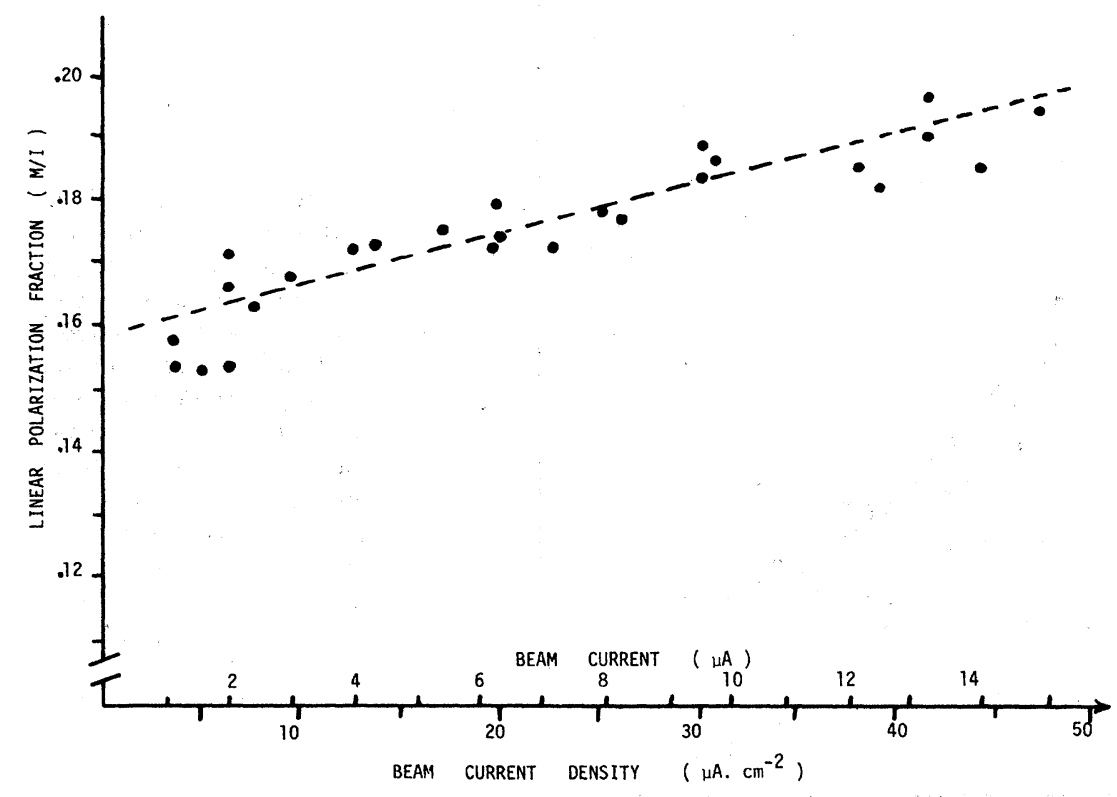

FIG. 1. Linear polarization $(M / I)$ of the $5016-\AA$, $2 s^{1} s-3 p{ }^{1} P$ He I transition excited by a $5 \pm 1-\mu \mathrm{g} \mathrm{cm}^{-2}$ carbon foil as a function of ion-beam current. The ion energy is $110 \mathrm{keV}$.

thermal properties were employed in the different experiments. Measurements were carried out using beams of different cross-sectional areas ( $\frac{3}{16}$ and $\frac{1}{4}$ in. diameter). Normalization was performed both to total beam current and total light intensity collected by an optical fiber bundle at a fixed distance from the foil. Agreement between the two sets of measurement in the region of overlap (100$180 \mathrm{keV}$ ) was excellent (see below).

\section{RESULTS}

Figure 1 illustrates the principal result obtained in this investigation: the dependence of $M / I$ upon ion current density $j$ for constant foil thickness. The figure shows a clear increase in $M / I$ with $j$ which is more marked at some energies than others. In order to investigate this energy dependence more quantitatively, we approximate the variation of the data with $j$ at each energy by a linear dependence (adequate within the statistical accuracy attained at all energies) and parametrize the rate of $j$ dependence of the polarization fraction $M / I$ by the slope of this curve, $S_{j}(E)$ for each ion energy $E$. Figure 2 then displays the complicated energy dependence of $S_{j}$. In the energy range studied, there are two maxima, one at approximately $100 \mathrm{keV}$ and

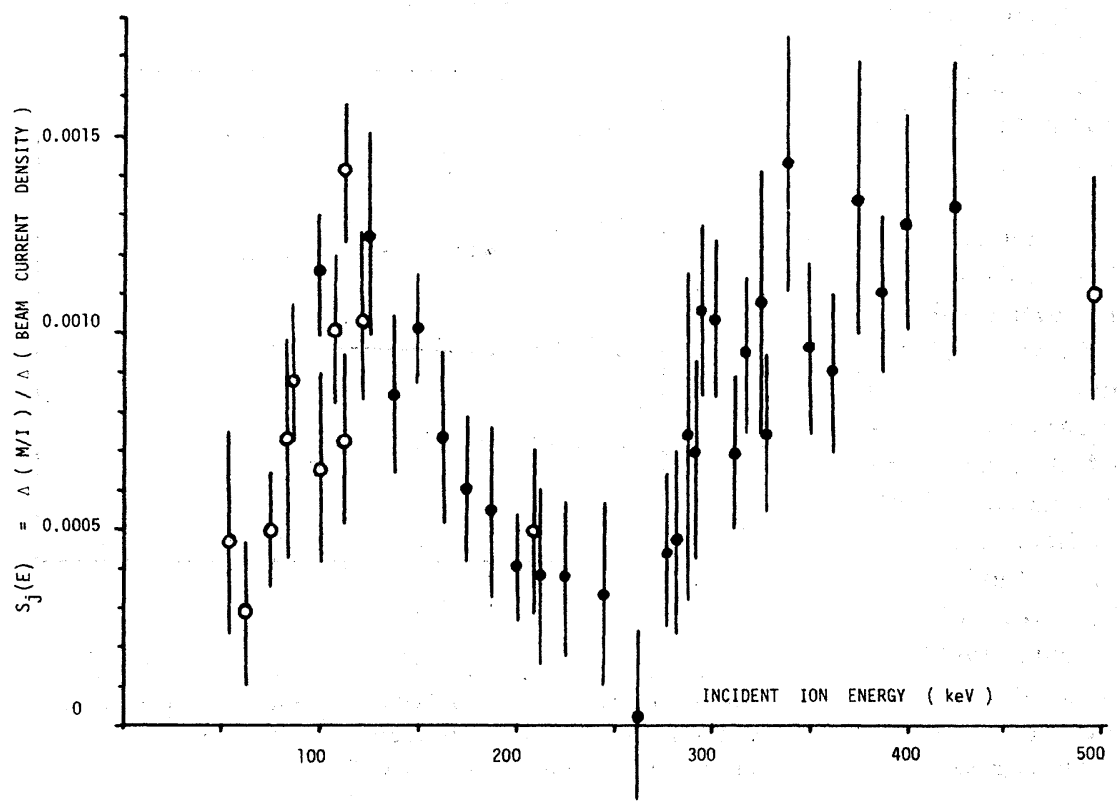

FIG. 2. Rate of change of linear polarization with beam-current density $S_{j}(E)$ as a function of the $\mathrm{He}^{+}$ion energy, for the 5016- $\AA$, $2 s^{1} S-3 p{ }^{1} P$ He I transition, excited by a $6 \pm 1-\mu \mathrm{g} \mathrm{cm}^{-2}$ carbon foil. The closed circles $(\bullet)$ are measurements at Toledo $(100-430$ $\mathrm{keV}$; the open circles (O) are measurements at Chicago $(50-200 \mathrm{keV})$ and Argonne (500 keV). 


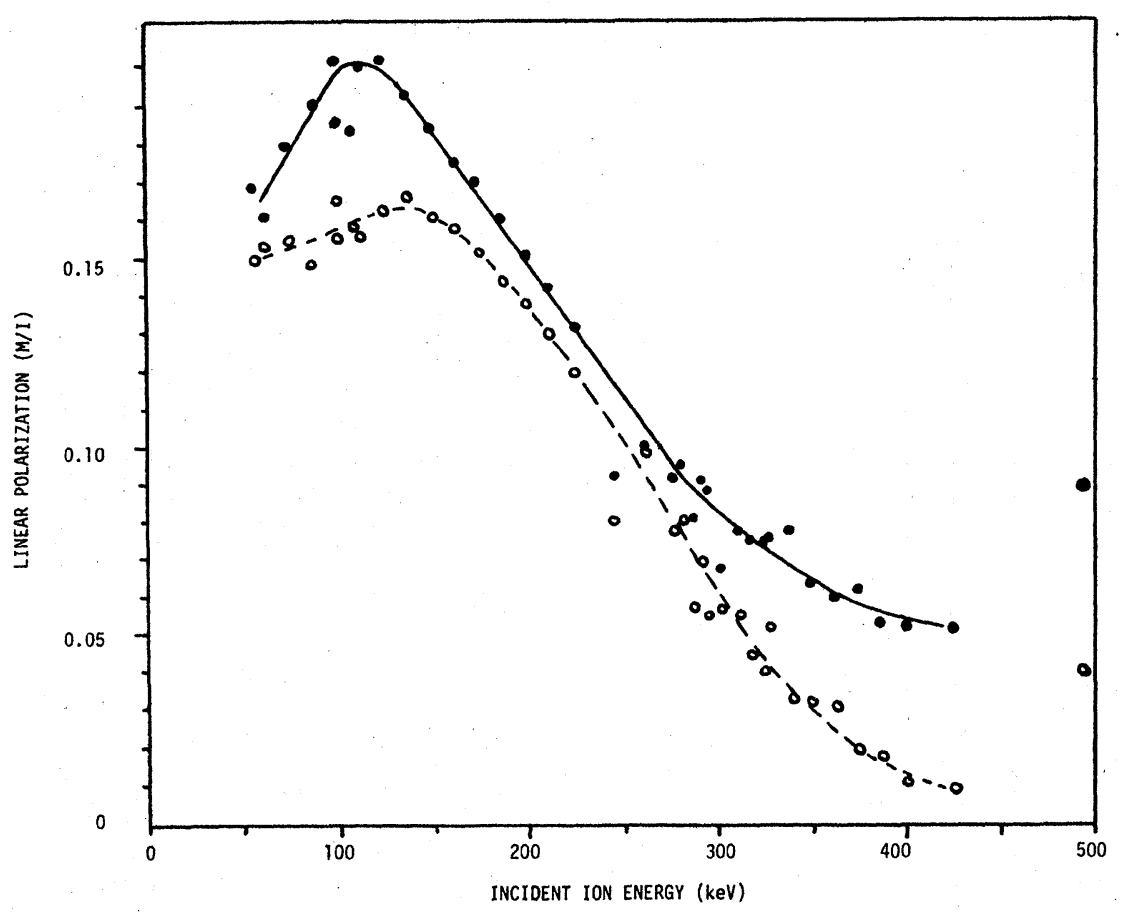

FIG. 3. Linear polarization fraction $(M / I)$ of the $5016-\AA, 2 s{ }^{1} S-3 p{ }^{1} P$ He I transition, excited by a $6 \pm 1-\mu \mathrm{g} \mathrm{cm}^{-2}$ carbon foil, as a function of ion energy. The closed circles $(\bullet)$ are from measurements made at beam current densities of 31 $\mu \mathrm{g} \mathrm{cm}^{-2}$. The open circles $(C)$ are extrapolations of our $M / I$ measurements to zero beam current.

a second near $400 \mathrm{keV}$. Less extensive measurements for the $2 p^{1} P-4 d^{1} D$ transition at $4922 \AA$ show a similar $j$ dependence of $M / I$, while measurements for $2 s{ }^{3} S-3 p{ }^{3} P$ at $3889 \AA$ show no such variations.

From the data described it is possible to construct curves showing the dependence of $M / I$ upon incident ion energy at constant beam-current density and foil thickness. The results for the $2 s{ }^{1} S-$ $3 p^{1} \mathrm{P}$ transition in HeI for beam current density extrapolated to zero and for $j=31 \mu \mathrm{A} / \mathrm{cm}^{2}$ are presented in Fig. 3. The confusion in interpretation of such measurements which can be caused by the current-density dependence is obvious and perhaps explains the discrepancy between these results and earlier investigations. ${ }^{4}$

We have also investigated the beam-current dependence of the alignment of the Ne II 3568- $\AA, 3 s^{\prime \prime}$ ${ }^{2} D_{5 / 2}-3 p^{\prime 2} F_{7 / 2}$ transition with thin carbon foil excitation at ion energies between 0.6 and $1.2 \mathrm{MeV}$. Figure 4 shows that within the small current range of $0.2-2.0 \mu \mathrm{A}\left(1-10 \mu \mathrm{A} \mathrm{cm}^{-2}\right)$, no variations outside statistics in $M / I$ could be detected, and $S_{j}(E)=0$. In addition, we carried out measurements of $M / I$ at constant incident-ion energy for a variety of foil thicknesses between 4 and $22 \mu \mathrm{g} / \mathrm{cm}^{2}$. Significant changes in $M / I$ were observed. However, within the statistical accuracy of these measurements, the observed charges can essentially be explained as effects due to the decrease in energy of the emergent beam with increasing foil thickness, in accord with an assumption of excitation equilibrium.

\section{DISCUSSION}

Since the origin of the alignment produced in the ion-foil interaction is not yet understood, it is especially difficult to establish the origin of the small changes in alignment with variation of beam current density reported here. Nevertheless, it is important to discuss possible causes in the hope

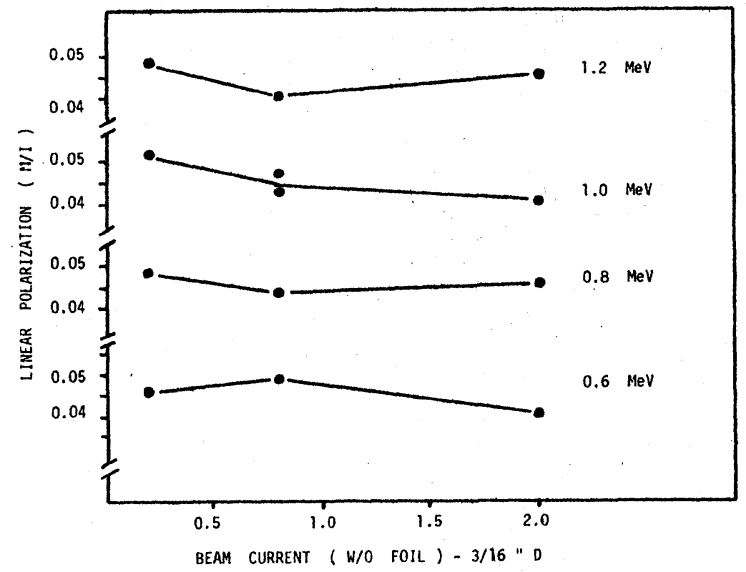

FIG. 4. Linear polarization fraction $(M / I)$ of the 3568- $\AA$ Ne II $3 s^{\prime} D_{5 / 2}=3 p^{\prime} F_{\eta / 2}$ transition excited by a $5 \pm 2-\mu \mathrm{g} \mathrm{cm}^{-2}$ carbon foil at ion energies of $0.6-1.2 \mathrm{MeV}$ and beam currents of $0.5-2.0 \mathrm{~A}\left(j=3-10 \mu \mathrm{A} \mathrm{cm}^{-2}\right)$. 
that this will suggest a better understanding of the basic phenomenon. It is helpful to divide the proposed mechanisms into microscopic and macroscopic effects.

\section{A. Microscopic effects}

We consider first possible variations in alignment with direct interparticle interactions in the beam. The average separation of particles $\langle d\rangle$ in the ion beam is determined from $\langle d\rangle=(q v / j)^{1 / 3}$. For a beam velocity $v$, ion charge $q$, and ion curent density $j$, typical parameters $\left(v=2.2 \times 10^{8}\right.$ $\mathrm{cm} \mathrm{s}^{-1}$ and $j=10 \mu \mathrm{Acm}^{-2}$ ) give a value $\langle d\rangle=0.14 \mathrm{~mm}$. Thus, direct interparticle interactions seem ruled out. On the other hand, indirect interactions are also possible. For example, each ion traversing the foil creates a wake ${ }^{13}$ or screening charge lagging behind the ion. Recently, Vager and Gemmell ${ }^{14}$ have been able to probe experimentally the shape and depth of the wake potential in observing molecular breakup in thin carbon foils. This polarization of the material will decay in times on the order of the inverse plasma frequency $\omega_{p}$ : that is, in about $10^{-14} \mathrm{~s}$. The time between successive ions passing through a cross-sectional area $A$ is $T=(q / j A)$. Considering the wake to have a width of $50 \AA$ (probably an upper limit ${ }^{14}$ ), we find that $T \approx 0.06 \mathrm{~s}$ for a current density of $10 \mu \mathrm{A} \mathrm{cm}^{-2}$. It is therefore also unlikely that a second beam particle can interact via this indirect mechanism. Similarly, dispersion of the "wake" polarization at the final surface will occur in about the same time, $\sim 10^{-14} \mathrm{~s}$, and the electric field or electron density at the surface, which will affect the final surface interaction with the beam ions, should be relatively independent of previous ion-induced surface polarization.

Trubnikov and Yablinskii ${ }^{15}$ investigated the pickup of conduction electrons by the moving ion as it leaves the surface. However, Cross ${ }^{16}$ has pointed out that the theoretical model used has an unrealistic cutoff in the conduction-electron distribution which leads to the wrong velocity dependence of the electron capture probability and also neglects electron capture from target atom cores in the surface. Nonetheless, Schröder and Kupfer ${ }^{9}$ have used the model of Ref. 15 to derive the alignment and orientation parameters of the hydrogen $2 p$ state produced in such an electron capture at a tilted surface. We have extracted from these calculations an expression for the linear polarization $(M / I)$ of a $p$ state, excited in our perpendicular foil geometry:

$$
M / I=(1-\eta) /(1+\eta),
$$

where

$$
\eta=\frac{E_{f}}{4 E_{e}} \frac{1}{1+n\left(E_{f}, E_{e}, W\right)} .
$$

$E_{f}$ is the Fermi energy, $E_{e}$ is the mean energy of a foil electron as viewed from the moving ion, and $n\left(E_{f}, E_{e}, W\right)$ is a complex function of these energies and the work function $W$ of the material, which varies relatively slowly. $E_{e}$ varies monotonically with ion energy, and this formula as it stands will not give rise to the oscillatory variations in $M / I$ or $S_{j}(E)$.

$E_{f}$ might be considered as a parameter, or effective Fermi energy, which is changed by increased ion current by, for example, increased secondary-electron production. Secondary electrons slowing down in the target can take sufficiently long to reach the final surface to change the parameters at the surface during excitation of a second ion.

A striking feature of our observations of $M / I$ and $S_{j}(E)$ is their oscillatory behavior with ion energy. Oscillations in cross sections have been observed in ion-atom collisions at much lower energy due to quantum-mechanical interference in the quasimolecular ion-atom system. ${ }^{17}$ The oscillations, whose frequencies are proportional to $1 / v$, have also been observed in the charge state fractions $N^{+} / N^{0}$ in ion-surface collisions at ion energies $1-10 \mathrm{keV}$. Tolk et al. ${ }^{18}$ postulate interferences in a quasimolecular state formed between the surface and the exiting atom. Unlike the ionatom collision in which specific molecular levels can be used to estimate the phase interference, the appropriate levels in the ion-solid interaction must be inferred from the experimental values of the oscillation frequencies.

Although polarization effects have not previously been measured in the ion-surface interaction, the ion-atom collisions show that the $1 / v$ oscillations occur most strongly in the parallel component of polarized light emitted from the excited atoms, but with large oscillations also in the polarization fraction $M / I$. It is thus tempting to compare our observed oscillation in $M / I$ with the ion-surface oscillations. If we assume two maxima at 100 and $400 \mathrm{keV}$ energy, we can evaluate the phase inte$\mathrm{gral}^{18}$

$$
\langle E R\rangle=\frac{0.5 h}{1 / v_{1}-1 / v_{2}},
$$

where $v_{1}, v_{2}$ are ion velocities, $h$ is Planck's constant and $\langle E R\rangle$ is the integral developed in the potential difference of two energy levels for internuclear separations $R$. By substitution, we find a value $\langle E R\rangle=34 \mathrm{eV} \AA$, which compares reasonably with values of about $20 \mathrm{eV} \AA$ found from ion-surface charge state oscillations. ${ }^{18}$ However, further maxima in $M / I$ should then occur at lower velocities, but none have been observed down to ion energies of $15 \mathrm{keV}$ [Tolk and Berry (unpublished)]. Other 
processes such as electron pickup may dominate $M / I$ production at these lower energies. It is difficult to associate the oscillations in $S_{j}(E)$ directly with this "Rosenthal" mechanism.

\section{B. Macroscopic effects}

The change in the linear polarization with beam current might be due to structural changes in the foil. However, we then observe that these changes must be reversible, since the $M / I$ values measured are independent of previous bombardment currents. The changes might be of two types: a change in the structure of the foil itself, for example, from a change of temperature (see below) or a change in the surface from changes in deposition and evaporation rates. In our vacuum of $5 \times 10^{-7}$ Torr, hydrocarbon buildup at the final surface is "cracked" by the ion-beam causing foil thickening at low beam currents. However, we have observe $d^{10}$ that this buildup rate is slow, with thicknesses sufficient to change $M / I$ after excitation by $\mathrm{Au}$ and $\mathrm{Ag}$ foils accumulating in times of $100-2000 \mathrm{~s}$. No changes in $M / I$ are found during a buildup on carbon foils. Nor is such a slow temporal change in $M / I$ observed when changing the beam current density. The changes occur within our time resolution of about $50 \mathrm{~s}$. Such reversible structural changes do not seem able to explain the variation of $M / I$ with beam current.

The foil temperature increases with increasing beam current. Also, increasing the foil thickness produces more energy loss in the foil and a consequent increase in temperature. We find that the energy deposited in the foil is lost radiatively at the surfaces, except for low beam currents (less than $1 \mu \mathrm{A}$ for a $5-\mu \mathrm{g} / \mathrm{cm}^{2}$ foil) where conduction losses become important. We have measured a foil temperature of $775 \pm 50^{\circ} \mathrm{C}$ with an optical pyrometer, compared to our calculated value of $740{ }^{\circ} \mathrm{C}$ for a beam current of $12 \mu \mathrm{A}$ of $110 \mathrm{keV} \mathrm{He}{ }^{+}$over a $\frac{3}{16}-$ in. -diam $22-\mu \mathrm{g} \mathrm{cm}^{-2}$ carbon foil, assuming an emissivity of 0.8 for the $C$ foil. The assumption that the equilibrium is determined by a balance between energy loss by the ion beam in the foil and heat dissipation mechanisms requires that identical changes in $M / I$ result from identical changes in $\Delta E$, the energy loss in the foil. However, for example, at $100 \mathrm{keV},-d E / d x$ is monotonically increasing ${ }^{19}$ and a small increase in the beam energy and a corresponding increase in the foil thickness should have identical effects upon $M / I$. This is in marked contrast with the data, suggesting that a simple temperature change, regardless of how it affects the $M / I$ production, is not correlated with the observed energy variation of $S_{j}(E)$.

\section{CONCLUSIONS}

We have measured the alignment of the $3 p{ }^{1} P$ state of HeI by observing the linear polarization $M / I$ of its decay to $2 s{ }^{1} S$ at $5016 \AA$ after excitation by thin carbon foils. We demonstrate that the alignment is a strong function of beam energy, showing oscillation, which we suggest might be due to quasimolecular ion-surface Rosenthal-type interfer ences. We cannot explain our observed variations of $M / I$ with beam current density $S_{j}(E)$. This parameter also shows oscillations with ion energy. We have discussed above some possible causes of these variations.

Similar polarization variations $S_{j}(E)$ are also observed for the $4922-\AA 2 p^{1} P-4 d^{1} D$ transition of $\mathrm{He} \mathrm{l,}$ but no beam-current-density variation $\left(S_{j}\right)$ could be found for the $3889 \AA 2 s{ }^{3} S-3 p{ }^{3} P$ HeI transitions at energies between 80 and $180 \mathrm{keV}$, nor for the $3568-\AA 3 s^{\prime 2} D_{5 / 2}-3 p^{\prime 2} F_{7 / 2} \mathrm{Ne}$ II transition between energies of 0.6 and $1.2 \mathrm{MeV}$.

\section{ACKNOWLEDGMENTS}

We thank Bob DeSerio, Gene Livingston, and Neal Schaffel for help in the measurements, and Jim Ray and Salvador Tejero for their technical help. Financial support from a Research Corpor ation grant (HGB) is gratefully acknowledged.

\footnotetext{
*Supported in part by the NSF and the U.S. ERDA.

${ }^{1}$ Beam Foil Spectroscopy (Proceedings of the Fourth International Conference), edited by Ivan A. Sellin and David J. Pegg (Plenum, New York, 1976); and references contained therein.

${ }^{2}$ H. G. Berry, Rep. Prog. Phys. 40, 155 (1977).

${ }^{3} \mathrm{~A}$ model of surface electric field production has been suggested by Eck (see Ref. 5) but does not seem to agree with the results of Denis et al., (Ref. 1, p. 799).

${ }^{4} \mathrm{An}$ extensive study of the energy-dependence alignment of the $n=2$ levels of $\mathrm{H}$ has been reported by $\mathrm{H}$. Winter and H. H. Bukow, Z. Phys. A 277, 27 (1976) extending the work of Dobberstein, Andrä, Wittmann and Bukow,
}

Z. Phys. 257, 272 (1972), and a preliminary measurement of the energy-dependence alignment of the $3 p{ }^{1} P$ level of He I has been presented by H. G. Berry and J. L. Subtil, Nucl. Instrum. Methods 110, 321 (1973).

${ }^{5}$ e.g. H. G. Berry, L. J. Curtis, D. G. Ellis, and R. M. Schectman, Phys. Rev. Lett. 32, 751 (1974); H. G. Berry, L. J. Curtis, D. G. Ellis, R. D. Hight, and R. M. Schectman, Ref. 1, p. 755.

${ }^{6}$ T. G. Eck, Phys. Rev. Lett. 33 , 1055 (1974); M. Lombardi, ibid. 35, 1172 (1975).

${ }^{7}$ Yehuda Band, Phys. Rev. Lett. 35, 1272 (1975).

${ }^{8}$ R. Herman, Phys. Rev. Lett. 35, 1626 (1975).

${ }^{9}$ H. Schröder and E. Kupfer, z. Phys. A 279, 13 (1976). 
${ }^{10}$ H. G. Berry, G. Gabrielse, T. Gay, and A. E. Livingston, Physica Scripta (to be published).

${ }^{i 1}$ U. Fano and J. Macek, Rev. Mod. Phys. 45, 553 (1973).

${ }^{12}$ H. G. Berry, G. Gabrielse, and A. E. Livingston, Appl. Opt. (to be published).

${ }^{13}$ See, e.g., J. Neufeld and R. H. Ritchie, Phys. Rev. 98, 1632 (1955); W. Brandt, in Atomic Collisions in Solids, edited by S. Datz, B. R. Appleton, and C. D. Moak (Plenum, New York, 1973), p. 621; W. Brandt and R. H. Ritchie, Nucl. Instrum. Methods 132, 43 (1976).

${ }^{14}$ Z. Vager and D. S. Gemmell, Phys. Rev. Lett. 37, 1352 (1976).
${ }^{15}$ B. A. Trubnikov and Y. M. Yablinskii, Zh. Eksp. Teor. Fiz. S2, 1638 (1967) [Sov. Phys,-JETP 25, 1089 (1967)], ${ }^{16}$ M. C. Cross, Phys. Rev. B 15, 602 (1977).

${ }^{17} \mathrm{H}$. Rosenthal and H. M. Foley, Phys. Rev. Lett. 23, 1480 (1969); N. A. Tolk, J. C. Tully, C. W. White, J. Kraus, A. Monge, D. L. Simms, M. F. Robbins, S. H. Neff, and W. Lichten, Phys. Rev. A 13, 969 (1976).

${ }^{18}$ R. L. Erickson and D. P. Smith, Phys. Rev. Lett. 34, 297 (1975); N. H. Tolk, J. C. Tully, J. Kraus, C. W. White, and S. H. Neff, Phys. Rev. A 13,969 (1976).

${ }^{19}$ L. C. Northcliffe and R. F. Schilling, Nucl. Data Tables A $\underline{7}, 233$ (1970). 